\title{
A comparative analysis of international health technology assessments for novel gene silencing therapies: patisiran and inotersen
}

\author{
Sergio lannazzo \\ Hetegy, Turin - Italy*
}

\begin{abstract}
Objectives: Using the case study of patisiran and inotersen, we conducted a narrative comparative analysis of the health technology assessment (HTA) agency appraisals of these two first-in-class transthyretin gene silencers, which represent exceptional advances in the treatment of hereditary transthyretin-mediated (hATTR) amyloidosis, a rare and multisystemic disease. Despite the impact of each product on the treatment landscape, the majority of HTAs are only considered standard of care as a comparator, resulting in a void of information and limited comprehension of the clinical and pharmacoeconomic differences between the two treatments.

Methods: A search was conducted internationally for HTA reports, and only instances where assessment decisions for both treatments were publicly available were included in the present analysis. The HTA reports were analyzed broadly for the assessment of clinical and pharmacoeconomic evidence. Only economic models considering both patisiran and inotersen were included in this analysis.

Results: A total of nine agencies with public assessment reports for both treatments were identified. HTA agency assessments for both treatments were essentially positive; however, differences were noted in the final recommendations, place in treatment or reimbursed indications, and in the narrative of the evaluations. Only the Canadian Agency for Drugs and Technologies in Health (CADTH) assessment for patisiran evaluated an economic model comparing the two treatments.

Conclusions: The differences summarized in this comparative analysis may provide a more comprehensive overview of the two treatments.
\end{abstract}

Keywords: hATTR, Health technology assessment, Hereditary transthyretin-mediated amyloidosis, Inotersen, ONPATTRO, Patisiran, TEGSEDI

\section{Introduction}

Hereditary transthyretin-mediated (hATTR) amyloidosis is a rare, multisystemic disease with a heterogeneous clinical presentation that impacts sensorimotor, autonomic, and cardiovascular function (1-4). Disease progression is rapid and leads to substantial functional disability, high morbidity, and mortality $(1,2)$. Historically, treatment options for hATTR amyloidosis were severely limited (1). Orthotopic liver transplantation (OLT) was an early treatment option and continues to be considered for a select proportion of early-stage polyneuropathy patients $(4,5)$. Previously, the

Received: October 23, 2020

Accepted: February 8, 2021

Published online: March 12, 2021

Corresponding author:

Sergio lannazzo

Via Cassini 19

10129 Turin - Italy

sergio.iannazzo@icloud.com

*Affiliation at the time of the study only pharmacological treatments available were transthyretin tetramer stabilizers that do not reduce the levels of the pathogenic protein. Multiple studies have shown that patients treated with these therapies experience slowing, but not halting, of neuropathy progression and declines in health-related quality of life (HRQoL) over time $(6,7)$.

Two first-in-class transthyretin (TTR) gene silencers for the treatment of hATTR amyloidosis received regulatory approval in multiple jurisdictions (8-11) and entered the reimbursement process in several countries at similar times. Patisiran (ONPATTRO $^{\circledR}$, Alnylam Pharmaceuticals), an RNA interference (RNAi) therapeutic, and inotersen (TEGSEDI ${ }^{\circledR}$, Akcea Therapeutics), an antisense oligonucleotide (ASO), represent a step change in therapy for hATTR amyloidosis patients and present promising treatment options for a population with substantial unmet need $(8,9)$.

As they are the first TTR gene silencers that inhibit the production of amyloid protein in hATTR amyloidosis, the clinical interest in their use is high. The review of two innovative orphan treatments for the same rare disease by health technology assessment (HTA) agencies at similar times is very uncommon, and even unprecedented under the Highly Specialised Technology (HST) program from the National Institute 
for Health and Care Excellence (NICE) (12). Given these points, these treatments are considered comparators; however, due to their entry in the reimbursement process at the same time, the majority of agencies reviewed each treatment in comparison to standard of care, which has resulted in a paucity of information on the clinical differences between the two treatments and a lack of comparative pharmacoeconomic evaluation. In this case study, we examine the conclusions of the HTA reports for patisiran and inotersen for hATTR amyloidosis with polyneuropathy to provide a comparative analysis for patients, clinicians, payers, and other stakeholders.

\section{Methods}

Published HTA reports were identified through a targeted literature search of international HTA agencies. HTA agencies were identified by searching the European Commission list of EU countries with an HTA system (13) and members of the International Network of Agencies for Health Technology Assessment (INAHTA) (14). Additional searches were conducted for major agencies not listed by either resource.

The initial search for published HTA appraisals was performed between July 1, 2019, and August 30, 2019, and was updated between November 12 and 20, 2020. The agency websites were searched using the following key words: patisiran, ONPATTRO, inotersen, and TEGSEDI. Public documents from HTA agencies were included for review if reports for patisiran and inotersen (separate or combined) were available. A narrative account of the assessment of the clinical and pharmacoeconomic evidence evaluated in the HTA report is presented, as well as the final agency recommendations for each treatment.

\section{Results}

Forty-four HTA agencies were identified internationally. Of these, nine HTA agencies had publicly available reports for patisiran and inotersen (Tab. I). While the majority of

TABLE I - HTA agencies with published appraisals for patisiran and inotersen*

\begin{tabular}{|c|c|}
\hline Country & Agency \\
\hline UK & $\begin{array}{l}\text { National Institute for Health and Care Excellence } \\
\text { (NICE) }\end{array}$ \\
\hline Scotland & Scottish Medicines Consortium (SMC) \\
\hline Canada & $\begin{array}{l}\text { Canadian Agency for Drugs and Technologies in } \\
\text { Health (CADTH) }\end{array}$ \\
\hline Germany & Gemeinsame Bundesausschuss (G-BA) \\
\hline France & Haute Autorité de Santé (HAS) \\
\hline Sweden & Tandvårds-och Läkemedelförmånsverket (TLV) \\
\hline United States & Institute for Clinical and Economic Review (ICER) \\
\hline Portugal & $\begin{array}{l}\text { Autoridade Nacional do Medicamento e } \\
\text { Produtos de Saúde (Infarmed) }\end{array}$ \\
\hline Spain & $\begin{array}{l}\text { Agencia Española de medicamentos y productos } \\
\text { sanitarios (AEMPS) }\end{array}$ \\
\hline
\end{tabular}

* Listed by descending publication date, ordered according to the first publication from the agency. the assessments contained evaluations of both the clinical and pharmacoeconomic evidence, the mandates of the HTA agencies included in this review varied in focus. Canadian Agency for Drugs and Technologies in Health (CADTH), Haute Autorité de Santé (HAS), the Gemeinsamer Bundesausschuss (G-BA), the Scottish Medicines Consortium (SMC), Agencia Española de medicamentos y productos sanitarios (AEMPS), Infarmed, and NICE play a formal role in the reimbursement process in their respective countries (15-23). Institute for Clinical and Economic Review (ICER) is an independent nonprofit organization that objectively evaluated the clinical and pharmacoeconomic value of patisiran and inotersen without providing a binding recommendation for payers in the United States (24). In Sweden, Tandvårdsoch Läkemedelsförmånsverket (TLV) provided pharmacoeconomic assessments of patisiran and inotersen at the request of the New Therapy Council, but did not provide a formal recommendation on reimbursement for either treatment (25-27).

The differences in the roles and mandates of the HTA agencies themselves are reflected in the structure and focus of the assessment reports. The summary results presented below are ordered chronologically by date of publication and are presented by agency. Within each agency heading, the conclusion or recommendation is reported first, followed by a review of the evaluation of the supporting clinical evidence, highlighting the differentiation in the treatment assessments. Language reported verbatim from the agency reports is indicated by quotation marks.

\section{National Institute for Health and Care Excellence}

Both patisiran and inotersen were recommended by NICE within their marketing authorizations $(15,20)$. The NICE appraisal for patisiran indicated there was evidence suggesting the drug may provide long-term benefits by stopping the progression of hATTR amyloidosis and potentially reversing the condition (20). Clinical experts consulted by NICE explained that "the likelihood of halting or reversing amyloid deposition, and so reducing neuropathy and improving cardiac function, is dependent on the extent of reduction in TTR" levels in the patient $(15,20)$. While the impact on the progression of the disease may vary among patients due to differences in production and turnover of amyloid in the body, the experts consulted believed that, although not validated, most patients would derive a clinically meaningful benefit from a TTR reduction of $80 \%$ or more (20). The NICE appraisal noted that in the patisiran group of the phase III trial, APOLLO, the mean reduction of serum TTR over 18 months was $87.8 \%$ (20). Additionally, the clinical experts believe that TTR reduction would be sustained over time with continued patisiran treatment because as TTR production is suppressed, the body is able to clear accumulated amyloid deposits (20). Therefore, the committee concluded that the clinical evidence from the APOLLO study showed that patisiran was of considerable benefit to patients and had the potential to stop and reverse disease progression (20). The inotersen group of the phase III NEURO-TTR trial showed a TTR reduction of $74 \%$ over 15 months (15). The committee concluded that while inotersen did not decrease serum TTR 
levels by $80 \%$, it provided a clinical benefit (15). The NICE appraisal reported that inotersen slowed the progression of neuropathy but did not stop progression or reverse the disease (15).

The NICE appraisal reported that patisiran reduced disability and improved HRQoL compared to baseline, which allowed patients to maintain their independence and dignity, resume or continue to carry out daily activities including returning to work, and participating in a more active social and family life (20). The NICE committee consulted patient experts who reported that the benefits seen in the APOLLO trial had "a marked effect on patients' lives, including regaining a social life, not having to wear incontinence pads, and being able to go to a restaurant without worrying about debilitating bowel symptoms." The NICE appraisal concluded that there was also evidence suggesting that patisiran may provide long-term HRQoL benefits to patients by stopping and potentially reversing the condition (20). Patient experts consulted during the NICE appraisal for inotersen indicated that slowing disease progression was valuable to them as it may allow them to remain in the earlier stages of disease, with a better quality of life, for longer (15).

The NICE appraisals for both patisiran and inotersen included assessment of the safety profiles of both therapies. The main safety concerns for inotersen highlighted by the NICE committee were related to glomerulonephritis and thrombocytopenia (15). One death in the inotersen arm, from intercranial hemorrhage caused by severe thrombocytopenia, was considered to be related to the study drug (15). After monitoring of platelet levels was implemented, no other severe thrombocytopenia events occurred in the NEURO-TTR trial, and the committee acknowledged that the major safety risks associated with inotersen can be effectively managed with routine monitoring in clinical practice (15). NICE reported the safety profile for patisiran was favorable, with a low stopping rate and no adverse events (AEs) that involved glomerulonephritis (20).

\section{Scottish Medicines Consortium}

The SMC assessment reports accepted patisiran and inotersen for use within NHS Scotland based on the substantial improvement in HRQoL associated with both treatments $(22,23)$

Clinical experts consulted by the SMC considered that patisiran represented a "major therapeutic advancement" and they noted that patisiran improved HRQoL and reduced disability compared to best supportive care (BSC) (22). The clinical experts consulted in the appraisal of inotersen considered it a "therapeutic advancement" that slowed the progression of polyneuropathy (23).

The SMC assessments included patient and clinical engagement (PACE) meetings consisting of patient group representatives and clinical specialists who considered the added value of the ultra-orphan drugs in the context of treatments currently available from NHS Scotland. The PACE meeting concluded that patisiran "has the potential to halt or slow progression of disease and in some cases improves symptoms," with the expectation of continued clinical improvement as long as TTR levels remain lowered (22). At the PACE meeting for inotersen, it was noted that compared to placebo, inotersen stabilizes progression of polyneuropathy and the corresponding decline in HRQoL (23).

\section{Canadian Agency for Drugs and Technologies in Health}

CADTH CDEC recommended based on separate assessments that both patisiran and inotersen be reimbursed for the treatment of polyneuropathy in adult patients with hATTR amyloidosis, under certain conditions (21). CADTH CDEC recognized that both patisiran and inotersen were associated with a statistically significant improvement compared with placebo for the primary and secondary endpoints of each pivotal study, respectively (21).

The CADTH report for patisiran included the result of an indirect treatment comparison (ITC) submitted by the manufacturer based on the two phase III studies APOLLO (patisiran) and NEURO-TTR (inotersen) (21). The ITC was conducted using two methods: one using the Bucher method and a second using the matching-adjusted indirect comparison (MAIC) method. Both analyses suggested that patisiran was statistically superior to inotersen for the change from baseline in modified Neuropathy Impairment Score $+7_{\text {ionis }}$ $\left(\mathrm{mNIS}+7_{\text {ionis }}\right)$ and the Norfolk Quality of Life Diabetic Neuropathy Instrument (Norfolk QoL-DN) scores (21). CADTH concluded that while the differences between treatments were statistically significant, the clinical significance of this was unclear, given the lack of published minimum clinical important difference (MCID) values for these outcome measures (21).

\section{Gemeinsamer Bundesausschuss}

The G-BA reported a "considerable additional benefit" for patisiran in the treatment of hATTR amyloidosis (19). This is the second highest possible designation from the G-BA, which corresponds to "a considerable improvement in therapy-related benefit, not previously achieved by BSC, such as recovery from disease, a considerable increase in life, longterm relief from severe symptoms, or extensive avoidance of severe side-effects" (28). The G-BA classified the extent of the additional benefit for inotersen as "not quantifiable" (18). This is the lowest possible designation by the G-BA for an orphan treatment, as an "additional benefit" is considered to be proven by the existence of the marketing authorization (28).

The G-BA assessed each study endpoint for the APOLLO and NEURO-TTR clinical trials in terms of statistical significance and clinical relevance. Only the endpoints that met both conditions were taken into consideration in the assessment of the additional therapeutic benefit for each treatment $(18,19)$. The endpoints considered as statistically significant and clinically relevant by the G-BA for patisiran included: gait speed (Ten Meter Walk Test; 10-MWT), health status (EuroQol 5 Dimension Visual Analog Scale; EQ-5D VAS), impairments regarding daily activities (Rasch-built Overall Disability Scale; R-ODS), and HRQoL (Norfolk QoL-DN) (19). These endpoints were regarded as a "considerable addition to the benefits" of patisiran even though the G-BA viewed their bias potential as high due to an imbalance in 
the number of missing observations for the placebo arm compared to the patisiran arm of APOLLO. The G-BA report explained that although the per-protocol treatment duration was 18 months for both the patisiran and the control arms, the mean treatment duration was longer for patisiran (18 months vs. 15 months), as there were more patients who discontinued in the placebo group (19). The G-BA assessment considered the majority of results from the NEURO-TTR trial "not quantifiable," with the exception of the physical functioning domain of the 36-Item Short Form Health Survey (SF-36) that measures HRQoL. A "positive effect" was reported for the SF-36, although the G-BA noted uncertainty regarding the possible overestimation of the effect (18).

The G-BA assessed the safety profile of both patisiran and inotersen. From the APOLLO trial, they noted that fewer AEs led to the discontinuation of treatment in the patisiran group than in the placebo group (19). Furthermore, there were more AEs in the placebo arm than in the patisiran arm. As the proportion of patients with at least 15 months treatment duration was higher in the patisiran arm than in the control arm, the G-BA reported the safety results from APOLLO were potentially biased to the disadvantage of patisiran (19). In the safety analysis of the NEURO-TTR trial, the G-BA noted that the higher level of AEs that resulted in discontinuation in the inotersen arm resulted in a "statistically significant disadvantage" for inotersen compared to placebo (18).

\section{Haute Autorité de Santé}

In their assessment of the place in therapy, HAS concluded patisiran should be considered a first-line treatment (16). The evaluation of inotersen by HAS concluded the drug should be considered a second-line treatment for patients who are unable to receive patisiran (17).

Neuropathy impairment was described as stabilized or improved at 18 months in the HAS appraisal for patisiran compared to baseline (29). The appraisal for inotersen described the treatment as demonstrating less neuropathy impairment than placebo at 15 months (30). The impact on HRQoL was described as "favorable" in the HAS appraisal for patisiran (29). In the appraisal for inotersen, the impact on HRQoL was described as "modest" (30).

When considering the safety profiles of both drugs, the HAS appraisal for patisiran reported an acceptable safety profile at 18 months that was characterized mainly by infusion-related reactions that were managed by premedication $(16,29)$. The HAS appraisal for inotersen noted the safety profile was characterized by the risk of thrombocytopenia that can be serious or even fatal-it was the cause of one death during the NEURO-TTR study, and the risk of glomerulonephritis which required additional renal function monitoring $(17,30)$. The inotersen appraisal also mentioned the existence of patisiran as an alternative treatment, with a safety profile that was considered to be more favorable in the short term $(17,30)$.

\section{Tandvårds-och Läkemedelsförmånsverket}

In their overall assessment of patisiran and inotersen, TLV made the assessment that "it is not possible to say that one drug is better than the other in terms of treatment effect" (31).
TLV reported that patisiran showed statistically significant and clinically relevant positive effects for the primary, secondary, and exploratory endpoints of the APOLLO, including patientreported outcomes (25). They further noted a sustained effect of patisiran in the long term based on the follow-up data to 36 months (25). The TLV analysis of inotersen reported statistically significant and clinically relevant positive effects for the primary composite endpoint from NEURO-TTR compared to placebo (26). TLV noted sustained efficacy for inotersen over the 15-month period of the NEURO-TTR study (26).

The TLV assessment of patisiran did not include any assessment or conclusion on the safety profile of patisiran (25). The assessment for inotersen indicated that while regular monitoring of platelet counts and renal and liver function is mandatory, there remains a risk of serious side effects with inotersen treatment compared to placebo (26).

\section{Institute for Clinical and Economic Review}

ICER reported a "moderate certainty of a substantial net health benefit with high certainty of at least a small net health benefit compared to best supportive care" for patisiran (24). As a result, they rated the net health benefit for patisiran as incremental or better than that of BSC, leading to a grade of $B+$ in the ICER evidence rating matrix (24). ICER reported a "moderate certainty of a comparable, small or substantial net health benefit for inotersen relative to BSC, with high certainty of at least a comparable net health benefit" (24). As a result, they rate the net health benefit for inotersen as comparable or better to that of BSC (ICER evidence rating matrix: $\mathrm{C}+)(24)$.

In their appraisal, ICER concluded that patisiran was associated with an improvement from baseline in neuropathy symptoms (24). Additionally, for the exploratory endpoint of neuropathy stage (disease progression), ICER noted that patients treated with patisiran were stable or improved compared to BSC in the APOLLO trial (24). The ICER appraisal of inotersen concluded that patients treated with the drug did not experience an improvement from baseline in neuropathy symptoms, but rather exhibited a slowing in worsening of neuropathy and stabilized neuropathy-related HRQoL relative to placebo (24). The report noted that the delay of neuropathy progression due to treatment with inotersen was sustained for 2 years; however, ICER indicated the stability in neuropathy-related HRQoL may not be durable. Additionally, ICER reported there was no evidence of improved stabilization of disease progression, as measured by polyneuropathy disability (PND) score, for patients treated with inotersen as compared to BSC (24).

ICER considered the safety profiles of both drugs and noted a "low-moderate" risk safety profile due to concomitant steroid administration for patisiran (24). Additionally, ICER reported a decreased frequency of AEs compared to BSC and no difference in mortality between treatment arms in APOLLO. As all deaths in the patisiran arm were cardiovascular in nature, a possible safety signal of complete heart block was reported; however, this finding was qualified by the statement that heart block can be observed in hATTR amyloidosis patients with cardiomyopathy. Finally, ICER noted that the post hoc analysis in patients with cardiac involvement demonstrated a statistically significant reduction in the 
composite rate of all-cause hospitalization and mortality in the patisiran arm compared to the BSC arm (24).

When considering the safety profile from the NEURO-TTR trial, ICER concluded that there were potential safety signals due to thrombocytopenia and glomerulonephritis in patients treated with inotersen (24). Uncertainty regarding the safety of inotersen treatment was reported given that all deaths in the NEURO-TTR trial occurred in the inotersen arm, and one patient death was considered possibly drug related. ICER suggested that the enhanced monitoring protocol added to the trial may offer assurance that the risk of thrombocytopenia may be manageable (24).

\section{Infarmed}

Infarmed recommended patisiran be reimbursed for the full indication in hATTR amyloidosis patients with stage 1 or stage 2 polyneuropathy (32). The report for inotersen recommended reimbursement for the treatment of patients with hATTR amyloidosis stage 2 polyneuropathy and patients in stage 1 who do not respond, who are intolerant, or ineligible for previous pharmacological treatment with tafamidis (33).

Both reports noted that while the magnitude of treatment effect was not possible to quantify, respectively, it may be considered substantial. The assessment of patisiran did not raise any safety concerns (32). The assessment of inotersen reported important toxicity problems that should be monitored (33).

\section{Agencia Española de medicamentos y productos sanitarios}

The AEMPS reports for patisiran and inotersen noted the lack of direct comparative studies between either treatment. Despite documenting the limitations of comparing the differences in endpoints ( $\mathrm{mNIS}+7 \mathrm{vs}$. $\mathrm{mNIS}+7_{\text {ionis }}$ ), the reports noted that an unadjusted indirect comparison showed an apparent greater effect of patisiran over inotersen on neuropathy $(34,35)$. The report for patisiran also noted a difference in safety profiles between the two treatments characterized by infusion reactions and ocular alterations for patisiran; and thrombocytopenia, risk of bleeding, and glomerulonephritis for inotersen (34).

\section{Limitations of the clinical evidence}

The limitations of clinical evidence raised in the HTA reports reviewed were similar for both treatments. In particular, the uncertainties surrounding long-term data for both patisiran and inotersen were mentioned by the majority of the HTA agencies (15-17,20-24,34-36). However, as noted in an earlier section, TLV reported a sustained effect of patisiran in the long term, based on the follow-up data to 36 months presented in the manufacturer's submission (25). TLV noted a sustained effect over the 15 months of the NEURO-TTR trial for patients treated with inotersen (26).

The clinical trial endpoints, mNIS+7 (patisiran, APOLLO) and $\mathrm{mNIS}+7_{\text {ionis }}$ (inotersen, NEURO-TTR), were criticized by some of the HTA agencies for the lack of published information on clinically relevant improvement $(16,17,21,24)$. CADTH, ICER, and HAS questioned the MCIDs of the mNIS+7 and $\mathrm{mNIS}+7_{\text {ionis }}$, while NICE, SMC, and TLV accepted the MCID of a 2-point change based on a consensus report from the
International Peripheral Nerve Society for the original NIS score $(15-17,20-26,36,37)$. Some HTA agency reports noted that neither $\mathrm{mNIS}+7$ scale is used in all clinical practice settings $(21,25,26,36)$. Additionally, the G-BA and HAS reported uncertainty associated with the appropriateness of the weights for each subcomponent of the scale (16-19). Finally, the imbalanced baseline characteristics between the drug and placebo groups were another source of uncertainty for both the APOLLO and NEURO-TTR trials $(15,19-21,24,26,36)$.

\section{Health economic components of the HTA appraisals}

NICE, the SMC, TLV, HAS, Infarmed, and the CADTH assessment of inotersen reviewed manufacturer-submitted pharmacoeconomic models in which patisiran or inotersen was compared to BSC in the base case $(15,20,22,23,29-33,36)$. The models for patisiran and inotersen had major methodological differences, including resource utilization, treatment costs, utilities, and health states, which inhibited comparison of the results. ICER developed separate Markov models for each treatment compared to BSC, as they indicated the differences in the primary outcome measures and trial populations precluded developing a pharmacoeconomic model comparing patisiran and inotersen (24). The Infarmed report for inotersen also included a subpopulation economic analysis comparing inotersen with tafamidis (33).

The CADTH appraisal of patisiran included a reanalysis of the manufacturer-submitted model that compared patisiran, inotersen, and BSC. The manufacturer's Markov model included health states defined by PND score and $\mathrm{N}$-terminal prohormone B-type natriuretic peptide (NT-proBNP) levels, OLT, and death. The CADTH reanalysis included modifications to harmonize the simulation of the clinical effects of patisiran, inotersen, and BSC, in order to enable a sequential cost-effectiveness analysis. The reanalysis also included an assumption of no difference in treatment-specific utilities and health state costs and removed the treatment effect of patisiran on cardiac outcomes. The CADTH reanalysis found that inotersen was extendedly dominated by the other treatments; in other words, inotersen was found less efficient than a combination of patisiran and BSC (21).

At the request of HAS, the manufacturer of patisiran submitted an additional comparison with inotersen as a scenario analysis; however, it was deemed inadequate and subsequently not considered in the assessment (29). The HAS evaluation of inotersen indicated that the manufacturer developed a comparison with patisiran, but did not submit it to the agency for assessment (30).

\section{Summary of the clinical and comparative economic evidence of the HTA appraisal}

The summary of the clinical and comparative pharmacoeconomic evidence from the HTAs reports is presented in Table II.

\section{Discussion}

When assessed individually, all HTA agencies highlighted areas of differentiation between the two treatments. While 
TABLE II - Summary of the clinical and comparative economic evidence of HTAs reviewed

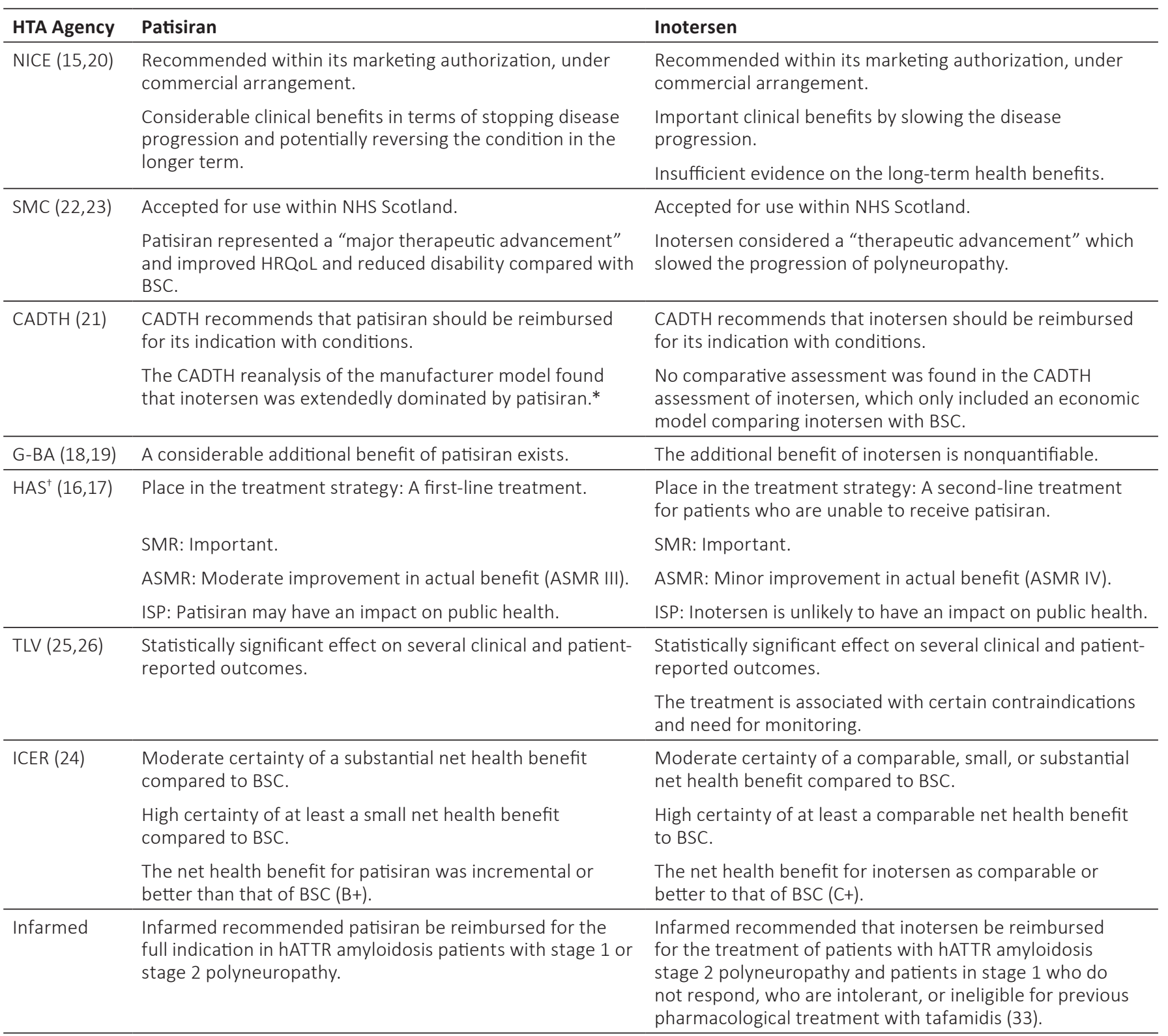

AEMPS AEMPS funded both treatments for their respective indications without restriction.

Both reports noted that an unadjusted indirect comparison showed an apparent greater effect of patisiran over inotersen on neuropathy.

The report for patisiran also noted a difference in safety profiles between the two treatments, characterized by infusion reactions and ocular alterations for patisiran; and thrombocytopenia, risk of bleeding, and glomerulonephritis for inotersen.

AEMPS = Agencia Española de medicamentos y productos sanitarios; BSC = best supportive care; CADTH = Canadian Agency for Drugs and Technologies in Health; G-BA = Gemeinsamer Bundesausschuss; HAS = Haute Autorité de santé; HRQoL = health-related quality of life; HTA = health technology assessment; ICER = Institute for clinical and economic review; ISP = Intérêt de santé publique (public health interest); NICE = National Institute for Health and Care Excellence; SMR = Service médical rendu (medical service rendered); TLV = Tandvårds-och Läkemedelsförmånsverket (The Dental and Pharmaceutical Benefits Agency).

${ }^{*}$ The CADTH appraisal of patisiran included a comparative economic analysis of patisiran, inotersen, and BSC. No other economic appraisals were reported as the results of the independent manufacturer models were not comparable due to methodological differences.

${ }^{+}$ASMR (Improvement in medical service rendered): ASMR I = majeure (major), ASMR II = importante (important), ASMR III = modérée (moderate), ASMR IV = mineure (minor), ASMR V = inexitante (inexistent). 
the HTA evaluations found both treatments improved the lives of patients, the majority acknowledged that the clinical evidence for patisiran demonstrated the potential to improve neuropathy symptoms, halt and reverse neuropathy progression, and maintain or improve patients' HRQoL $(16,20,21,24)$. Inotersen was described in many of the HTA appraisals as having the ability to stabilize or slow the decline in neuropathy symptoms, delay neuropathy progression, and slow the decline of HRQoL in hATTR amyloidosis patients $(15,17,24,35)$.

The distinctions between the two treatments are further supported by the place in therapy assessments by HAS, which recommended patisiran as a first-line treatment and inotersen as a second-line treatment for patients who were not able to receive patisiran $(16,17)$. The AEMPS assessment for patisiran reported an apparent greater effect of patisiran over inotersen; however, they acknowledged that this could not be confirmed due to limitations in the indirect and unadjusted comparison (34). ICER and the G-BA use a scale or matrix rating system in their appraisals and both agencies rated patisiran higher than inotersen using their respective rating systems $(18,19,24)$.

While no direct head-to-head trials exist to compare patisiran and inotersen, the aspects of clinical differentiation reported by the HTA appraisals considered in this case study were supported by the findings of the ITC adopted as part of the CADTH recommendation for patisiran. The results of the ITC found a statistically significant difference between treatments; however, CADTH also concluded that due to the lack of published MCID for the outcomes measured, the clinical significance of the difference was unclear (21).

The safety profiles for both patisiran and inotersen were reviewed by all agencies. Almost all assessments reported that the safety profile for patisiran was acceptable (16, 19-21,25,32-36). The ICER appraisal included a recommendation of a potential safety signal for complete heart block. However, the agency acknowledged that heart block is observed as a common symptom of cardiac involvement in hATTR amyloidosis (24).

Several appraisals reported AEs of glomerulonephritis and thrombocytopenia for inotersen in the NEURO-TTR trial, which resulted in enhanced monitoring of trial patients $(15,17,18,24,26,33,35,36)$. TLV expressed concern with the safety profile for inotersen despite the additional monitoring (26).

While the HTA economic conclusions were difficult to compare, CADTH performed a reanalysis of the patisiran manufacturer model that included three comparators (patisiran, inotersen, and BSC). The CADTH model reported a sequential analysis that showed inotersen was less efficient than a combination of patisiran and BSC (21).

The HTA reports included in this case study concluded that both patisiran and inotersen have the ability to improve patients' lives. The clinical and pharmacoeconomic differences reported in the assessments may provide a useful overview for those seeking a more comprehensive understanding of the two treatments and may be of assistance to relevant stakeholders when considering treatment options for hATTR amyloidosis.

\section{Acknowledgments}

The author wishes to thank Véronique Lauzon, MSc and Nicole Tunstall, MSc of International Market Access Consulting $\mathrm{GmbH}$, for providing medical writing support.

\section{Disclosure}

Conflict of interest: Dr. lannazzo reports personal fees from Alnylam Pharmaceuticals.

Financial support: This study was supported by Alnylam Pharmaceuticals.

\section{References}

1. Adams D, Suhr OB, Hund E, et al; European Network for TTR-FAP (ATTReuNET). First European consensus for diagnosis, management, and treatment of transthyretin familial amyloid polyneuropathy. Curr Opin Neurol. 2016;29(suppl 1):S14-S26. CrossRef PubMed

2. Ando Y, Coelho T, Berk JL, et al. Guideline of transthyretinrelated hereditary amyloidosis for clinicians. Orphanet J Rare Dis. 2013;8(1):31. CrossRef PubMed

3. Gertz MA. Hereditary ATTR amyloidosis: burden of illness and diagnostic challenges. Am J Manag Care. 2017;23(7) (suppl):S107-S112. PubMed

4. Hawkins PNAY, Ando $Y$, Dispenzeri A, Gonzalez-Duarte A, Adams $D$, Suhr OB. Evolving landscape in the management of transthyretin amyloidosis. Ann Med. 2015;47(8):625-638. CrossRef PubMed

5. Carvalho A, Rocha A, Lobato L. Liver transplantation in transthyretin amyloidosis: issues and challenges. Liver Transpl. 2015;21(3):282-292. CrossRef PubMed

6. Barroso FAJD, Judge DP, Ebede $B$, et al. Long-term safety and efficacy of tafamidis for the treatment of hereditary transthyretin amyloid polyneuropathy: results up to 6 years. Amyloid. 2017;24(3):194-204. CrossRef PubMed

7. Berk JL. TTR Gene silencers for hereditary ATTR amyloidosis: more than ICER recognized. J Manag Care Spec Pharm. 2019;25(1):15-16. CrossRef PubMed

8. European Medicines Agency. (2018). Onpattro (patisiran). Online Accessed July 5, 2019.

9. European Medicines Agency. (2018). Tegsedi (inotersen). Online Accessed July 5, 2019.

10. Ledford H. Gene-silencing technology gets first drug approval after 20-year wait. Nature. 2018;560(7718):291-292. CrossRef PubMed

11. U.S. Food \& Drug Administration. (2018). Drug Approval Package: Tegsedi (inotersen). U.S. Food \& Drug Administration. (2018). Drug Approval Package: Tegsedi (inotersen). Online Accessed July 9, 2019.

12. National Institute for Health and Care Excellence. NICE Guidance and advice list. Online Accessed September 6, 2019.

13. European Commission. (2017). Mapping of HTA national organisations, programmes and processes in EU and Norway 1-51. Online Accessed July 9, 2019.

14. INAHTA. (2019). The International Network of Agencies for Health Technology Assessment. Online Accessed July 8, 2019.

15. National Institute for Health and Care Excellence. (2019). Final evaluation document: Inotersen for treating hereditary transthyretin-related amyloidosis 1-24. Online Accessed July 9, 2019.

16. Commission de la transparence. (2019). Avis 20 mars 2019, patisiran 1-21. Online Accessed July 9, 2019.

17. Commission de la transparence. (2019). Avis 17 avril 2019, inotersen 1-23. Online Accessed July 9, 2019. 
18. Gemeinsamer Bundesausschuss. (2019). Tragende Gründe zum Beschluss des Gemeinsamen Bundesausschusses über eine Änderung der Arzneimittel-Richtlinie (AM-RL): Anlage XII-Beschlüsse über die Nutzenbewertung von Arzneimitteln mit neuen Wirkstoffen nach § 35a SGB V-Inotersen 1-14. Online Accessed July 9, 2019.

19. Gemeinsamer Bundesausschuss. (2019). Tragende Gründe zum Beschluss des Gemeinsamen Bundesausschusses über eine Änderung der Arzneimittel-Richtlinie (AM-RL): Anlage XII-Beschlüsse über die Nutzenbewertung von Arzneimitteln mit neuen Wirkstoffen nach § 35a SGB V-Patisiran 1-15. Online Accessed July 9, 2019.

20. National Institute for Health and Care Excellence. (2019). Final evaluation document: Patisiran for treating hereditary transthyretin amyloidosis 1-32. Online Accessed July 9, 2019.

21. Canadian Agency for Drugs and Technologies in Health. (2019). CADTH Canadian Drug Expert Committee Recommendation. Patisiran (ONPATTRO-Alnylam Netherlands BV). Online Accessed August 5, 2019.

22. Scottish Medicines Consortium. (2019). SMC Advice on new medicines: patisiran $2 \mathrm{mg} / \mathrm{mL}$ concentrate for solution for infusion (Onpattro ${ }^{\circledR}$ ). Online Accessed July 8, 2019.

23. Scottish Medicines Consortium. (2019). SMC advice on new medicines: inotersen $284 \mathrm{mg}$ solution for injection in pre-filled syringe (Tegsedi ${ }^{\circledR}$ ). Online Accessed August 20, 2019.

24. Institute for Clinical and economic Review. Inotersen and Patisiran for Hereditary Transthyretin Amyloidosis: Effectiveness and Value (2018) i-153. Online Accessed July 9, 2019.

25. Tandvards-OCH Lakemedelsformansverket. (2018). Underlag för beslut i landstingen: Onpattro (patisiran) i-43. Online Accessed July 9, 2019.

26. Tandvards-OCH Lakemedelsformansverket. (2019). Underlag för beslut i landstingen: Tegsedi (inotersen) i-31. Online Accessed July 9, 2019.

27. The New Therapies Council. Managed introduction-this is how it works. Online Accessed July 26, 2019.
28. Wenzl M, Paris V. (June 2018). Pharmaceutical reimbursement and pricing in Germany. In OECD (Ed.), 1:22. Online Accessed July 9, 2019.

29. Haute Autorité de Santé. (2019). Avis d'Efficience. Onpattro (patisiran) Amylose héréditaire à transthyrétine chez les patients adultes atteints de polyneuropathie de stade 1 ou de stade 2. Online Accessed July 8, 2019.

30. Haute Autorité de Santé. (2019). Avis d’Efficience. Tegsedi (inotersen) Amylose à transthyrétine héréditaire chez les patients adultes atteints de polyneuropathie de stade 1 ou de stade 2. Online Accessed July 8, 2019.

31. Tandvards-OCH Lakemedelsformansverket. (2019). Underlag för beslut i regionerna En samlad bedömning av Onpattro och Tegsedi. Online Accessed July 8, 2019.

32. Infarmed Autoridade Nacional do Medicamento e Produtos de Saúde L.P. Relatório público de avalição: ONPATTRO (patisiran) 1-65. Online Accessed November 20, 2020.

33. Infarmed Autoridade Nacional do Medicamento e Produtos de Saúde L.P. Relatório. Online Accessed November 20, 2020.

34. Agencia española de medicamentos y productos sanitarios. (2020). Informe de Posicionamiento Terapéutico de patisirán (Onpattro ${ }^{\circledR}$ ) en el tratamiento de la amiloidosis hereditaria por transtiretina 1-7. Online Accessed November 20, 2020.

35. Agencia española de medicamentos y productos sanitarios. (2020). Informe de Posicionamiento Terapéutico de inotersén $\left(\right.$ Tegsedi $^{\circledR}$ ) en el tratamiento de la amiloidosis hereditaria por transtiretina 1-9. Online Accessed November 20, 2020.

36. Canadian Agency for Drugs and Technologies in Health. (2019). CADTH Canadian Drug Expert Committee Recommendation: inotersen (TEGSEDI, Akcea Theapeutics, Inc.). Online Accessed November 14, 2020.

37. International Peripheral Nerve Society. Diabetic polyneuropathy in controlled clinical trials: Consensus Report of the Peripheral Nerve Society. Ann Neurol. 1995;38(3):478-482. CrossRef PubMed 